\title{
A Hybrid Power Mean Involving the Dedekind Sums and Cubic Gauss Sums
}

\author{
Jiayuan $\mathrm{Hu} \mathbb{D}^{1},{ }^{1}$ Yu Zhan, ${ }^{2}$ and Qin $\mathrm{Si}^{1}$ \\ ${ }^{1}$ Department of Mathematics and Computer Science, Hetao College, Bayannur, China \\ ${ }^{2}$ Department of Civil Engineering, Hetao College, Bayannur, China \\ Correspondence should be addressed to Jiayuan Hu; hujiayuan1986@163.com
}

Received 11 August 2021; Revised 23 August 2021; Accepted 4 October 2021; Published 18 October 2021

Academic Editor: Yuan Yi

Copyright (c) 2021 Jiayuan Hu et al. This is an open access article distributed under the Creative Commons Attribution License, which permits unrestricted use, distribution, and reproduction in any medium, provided the original work is properly cited.

The main purpose of this paper is using analytic methods and the properties of the Dedekind sums to study one kind hybrid power mean calculating problem involving the Dedekind sums and cubic Gauss sum and give some interesting calculating formulae for it.

\section{Introduction}

For any integer $q \geq 3$ and integer $m$, the classical cubic Gauss sum $A(m, q)$ is defined as follows:

$$
A(m, q)=\sum_{a=0}^{q-1} e\left(\frac{m a^{3}}{q}\right)
$$

where as usual, $e(x)=e^{2 \pi i x}$ and $i^{2}=-1$.
This sum plays a very important role in the study of the elementary number theory and analytic number theory, so there are many people who had studied the arithmetical properties of $A(m, q)$ and related contents (see [1-6]). For example, if $q=p$ is an odd prime, Cao and Wang [5] proved the following interesting conclusion: let $p$ be an odd prime with $p \equiv 1 \bmod 3$. If 3 is not a cubic residue modulo $p$, then we have the identity

$$
\sum_{m=1}^{p-1}\left|\sum_{a=0}^{p-1} e\left(\frac{m a^{3}}{p}\right)\right|^{2} \cdot\left|\sum_{a=0}^{p-1} e\left(\frac{m a^{3}+a}{p}\right)\right|^{4}=p^{2}\left(4 p^{2}-19 p-d^{2}-1\right)
$$

where $4 p=d^{2}+27 \cdot b^{2}$ and $d$ is uniquely determined as follows: $d \equiv 1 \bmod 3$

On the other hand, we define the Dedekind sums $S(h, q)$ as follows.

Let $q$ be a natural number and $h$ be an integer prime to $q$. The classical Dedekind sum $S(h, q)$ is defined as

$$
S(h, q)=\sum_{a=1}^{q}\left(\left(\frac{a}{q}\right)\right)\left(\left(\frac{a h}{q}\right)\right)
$$

where

$$
((x))= \begin{cases}x-[x]-\frac{1}{2}, & \text { if } x \text { is not an integer } \\ 0, & \text { if } x \text { is an integer. }\end{cases}
$$

About the various properties of $S(h, q)$, many authors had studied them and obtained a series of interesting results, and related works can be found in [7-14].

The main purpose of this paper is using the analytic method and the properties of Dedekind sums to study the computational problem of the hybrid power mean: 


$$
\frac{\sum_{m=1}^{q^{\prime}} S^{h}(m, q)}{A^{k}(m, q)}
$$

and give some exact computational formulae for (5) with $q=p$, an odd prime, where $h$ and $k$ are any two fixed positive integers.

About this problem, so far no one seems to consider; at least, we have not seen any related papers before. Of course, this problem is meaningful, and it can describe the mean value distribution properties of the two different sums. It is clear that if $(p-1,3)=1$, then from the properties of the complete residue system modulo $p$, we have $A(m)=0$. This time (5) is meaningless. So, in the following, we only consider the case $p \equiv 1 \bmod 3$. The main purpose of this paper is to prove the following several results.

Theorem 1. Let $p$ be a prime with $p=12 h+1$. Then, for any positive integers $k$ and $h$ with $(2, h)=1$, we have the identity

$$
\sum_{m=1}^{p-1} \frac{S\left(m^{2}, p\right)}{A^{k}(m, p)}=\sum_{m=1}^{p-1} \frac{S^{h}(m, p)}{A^{k}(m, p)}=0 .
$$

Theorem 2. Let $p$ be a prime with $p=12 h+7$. Then, for any positive integer $k$, we have the identity

$$
\sum_{m=1}^{p-1} \frac{S\left(m^{2}, p\right)}{A^{k}(m, p)}=\frac{1}{\pi^{2}} \cdot \frac{p}{p-1} \cdot\left(\frac{p-1}{A^{k}(1, p)}-H(k, p)\right) \cdot\left|L\left(1, \chi_{2} \lambda\right)\right|^{2}+\frac{h_{p}^{2}}{p-1} \cdot H(k, p),
$$

where $\chi_{2}$ is Legendre's symbol modulo $p, \lambda$ denotes any thirdorder character modulo $p, h_{p}=\sqrt{p} / \pi \cdot\left|L\left(1, \chi_{2}\right)\right|$ denotes the class number of the quadratic field $F_{p}(\sqrt{-p})$, and

$$
H(k, p)=\sum_{m=1}^{p-1} \frac{1}{A^{k}(m, p)},
$$

satisfies the third-order linear recursive formula as follows:

$$
H(k, p)=-\frac{3}{d} H(k-1, p)+\frac{1}{d p} H(k-3, p),
$$

with the initial values $H(0, p)=p-1, H(1, p)=-p-1 / d$, and $H(2, p)=3(p-1) / d^{2}$, and $4 p=d^{2}+27 \cdot b^{2}$, where $d$ is uniquely determined by $d \equiv 1 \bmod 3$.

Theorem 3. Let $p$ be a prime with $p \equiv 1 \bmod 3$. Then, for any positive integer $k$, we have the asymptotic formula as follows:

$$
\sum_{m=1}^{p-1} \frac{S^{2}(m, p)}{A^{k}(m, p)}=\left(\frac{5 p}{144}+\frac{p}{\pi^{4}} \cdot \sum_{n=1}^{\infty} \frac{\left|\sum_{d \mid n} \lambda(d)\right|^{2}}{n^{2}}\right) \cdot H(k, p)+O\left(\frac{p}{|d|^{k}} \cdot \exp \left(\frac{4 \ln p}{\ln \ln p}\right)\right)
$$

where $\exp (y)=e^{y}$ and $\lambda$ is a three-order character modulo $p$.

Corollary 1. Let Let $p$ be a prime with $p \equiv 1 \bmod 3$, then we

From Theorem 3, we may immediately deduce the folhave lowing two corollaries.

$$
\sum_{m=1}^{p-1} \frac{S^{2}(m, p)}{A(m, p)}=-\frac{p^{2}}{d} \cdot\left(\frac{5}{144}+\frac{1}{\pi^{4}} \cdot \sum_{n=1}^{\infty} \frac{\left|\sum_{d \mid n} \lambda(d)\right|^{2}}{n^{2}}\right)+O\left(\frac{p}{|d|} \cdot \exp \left(\frac{4 \ln p}{\ln \ln p}\right)\right) .
$$

Corollary 2. Let $p$ be a prime with $p \equiv 1 \bmod 3$, then we have

$$
\sum_{m=1}^{p-1}\left|\frac{S(m, p)}{A(m, p)}\right|^{2}=\frac{3 p^{2}}{d^{2}} \cdot\left(\frac{5}{144}+\frac{1}{\pi^{4}} \sum_{n=1}^{\infty} \frac{\left|\sum_{d \mid n} \lambda(d)\right|^{2}}{n^{2}}\right)+O\left(\frac{p}{d^{2}} \cdot \exp \left(\frac{4 \ln p}{\ln \ln p}\right)\right) .
$$




\section{Several Lemmas}

To complete the proofs of our all theorems, we need to prove several simple lemmas. Hereinafter, we shall use some properties of the character sums and Gauss sums, and all of these contents can be found in [15], so they will not be repeated here.

Lemma 1. Let $p$ be an odd prime with $p \equiv 1 \bmod 3$ and $\lambda$ be any third-order character modulo $p$, then we have the identity

$$
\tau^{3}(\lambda)+\tau^{3}(\bar{\lambda})=d p,
$$

where $\tau(\lambda)=\sum_{a=1}^{p-1} \lambda(a) e(a / p)$ denotes the classical Gauss sums, and $4 p=d^{2}+27 \cdot b^{2}$, where $d$ is uniquely determined by $d \equiv 1 \bmod 3$.

Proof. The proof of this lemma is shown in the study by Zhang and $\mathrm{Hu}[2]$ or Berndt and Evans [16].
Lemma 2. Let $p$ be an odd prime with $p \equiv 1 \bmod 3$. Then, for any integer $k \geq 3$ and

$$
H(k, p)=\sum_{m=1}^{p-1} \frac{1}{A^{k}(m, p)},
$$

we have the third-order linear recursive formula as follows:

$$
H(k, p)=-\frac{3}{d} H(k-1, p)+\frac{1}{d p} H(k-3, p),
$$

with the initial values $H(0, p)=p-1, H(1, p)=-p-1 / d$, and $H(2, p)=3(p-1) / d^{2}$, where $d$ is defined as in Lemma 1.

Proof. Let $\lambda$ be any third-order character modulo $p$; then, for any integer $1 \leq m \leq p-1$, from the properties of the third-order characters and the classical Gauss sums modulo $p$, we have the identity

$$
\begin{aligned}
A(m, p) & =\sum_{a=0}^{p-1} e\left(\frac{m a^{3}}{p}\right)=1+\sum_{a=1}^{p-1}(1+\lambda(a)+\bar{\lambda}(a)) e\left(\frac{m a}{p}\right), \\
& =\sum_{a=0}^{p-1} e\left(\frac{m a}{p}\right)+\bar{\lambda}(m) \tau(\lambda)+\lambda(m) \tau(\bar{\lambda})=\bar{\lambda}(m) \tau(\lambda)+\lambda(m) \tau(\bar{\lambda}) .
\end{aligned}
$$

Note that $\tau(\lambda) \tau(\bar{\lambda})=p$ and $\lambda^{2}=\bar{\lambda}$; from (16), we have

From (16) and Lemma 1, we also have

$$
A^{2}(m, p)=\lambda(m) \tau^{2}(\lambda)+2 p+\bar{\lambda}(m) \tau^{2}(\bar{\lambda}) .
$$

$$
\begin{aligned}
A^{3}(m, p) & =(\bar{\lambda}(m) \tau(\lambda)+\lambda(m) \tau(\bar{\lambda}))^{3} \\
& =\tau^{3}(\lambda)+\tau^{3}(\bar{\lambda})+3 p A(m, p)=d p+3 p A(m, p) .
\end{aligned}
$$

From (17) and the properties of the character sums modulo $p$, we have

$$
\sum_{m=1}^{p-1} A^{2}(m, p)=\sum_{m=1}^{p-1}\left(\lambda(m) \tau^{2}(\lambda)+2 p+\bar{\lambda}(m) \tau^{2}(\bar{\lambda})\right)=2 p(p-1)
$$

Combining (18) and (19), we can deduce that

$$
\sum_{m=1}^{p-1} A^{2}(m, p)=d p \cdot \sum_{m=1}^{p-1} \frac{1}{A(m, p)}+3 p \sum_{m=1}^{p-1} 1
$$

$$
\sum_{m=1}^{p-1} \frac{1}{A(m, p)}=\frac{1}{d p}(2 p(p-1)-3 p(p-1))=-\frac{p-1}{d}
$$

Similarly, from (16), (18), and (21), we have 


$$
0=\sum_{m=1}^{p-1} A(m, p)=\sum_{m=1}^{p-1} \frac{d p}{A^{2}(m, p)}+\sum_{m=1}^{p-1} \frac{3 p}{A(m, p)}=\sum_{m=1}^{p-1} \frac{d p}{A^{2}(m, p)}-\frac{3 p(p-1)}{d}
$$

or

$$
\sum_{m=1}^{p-1} \frac{1}{A^{2}(m, p)}=\frac{3(p-1)}{d^{2}}
$$

If $k \geq 3$, then from (18), we have

$$
\frac{1}{A^{k-3}(m, p)}=d p \cdot \frac{1}{A^{k}(m, p)}+3 p \cdot \frac{1}{A^{k-1}(m, p)}
$$

or

$$
H(k, p)=-\frac{3}{d} H(k-1, p)+\frac{1}{d p} H(k-3, p) .
$$

Now, Lemma 2 follows from (21)-(25) and $H(0, p)=p-1$.
Lemma 3. Let $p$ be a prime with $p \equiv 1 \bmod 3$. For any positive integer $k$ and any character $\chi \bmod p$, if $\chi^{3} \neq \chi_{0}$, then we have the identity

$$
\sum_{m=1}^{p-1} \frac{\chi(m)}{A^{k}(m, p)}=0
$$

where $\chi_{0}$ denotes the principal character modulo $p$.

Proof. Let $g$ be a primitive root modulo $p$. Then, from the definition of $A(m, p)$ and the properties of the complete residue system modulo $p$, we have

$$
A\left(m g^{3}, p\right)=\sum_{a=0}^{p-1} e\left(\frac{m g^{3} a^{3}}{p}\right)=\sum_{a=0}^{p-1} e\left(\frac{m(g a)^{3}}{p}\right)=\sum_{a=0}^{p-1} e\left(\frac{m a^{3}}{p}\right)=A(m, p)
$$

From (27), we have

$$
\sum_{m=1}^{p-1} \frac{\chi(m)}{A^{k}(m, p)}=\sum_{m=1}^{p-1} \frac{\chi\left(m g^{3}\right)}{A^{k}\left(m g^{3}, p\right)}=\chi^{3}(g) \cdot \sum_{m=1}^{p-1} \frac{\chi(m)}{A^{k}(m, p)} .
$$

or

$$
\left(1-\chi^{3}(g)\right) \cdot \sum_{m=1}^{p-1} \frac{\chi(m)}{A^{k}(m, p)}=0 .
$$

If $\chi^{3} \neq \chi_{0}$, then $\chi^{3}(g) \neq 1$. So, from (29), we have the identity

$$
\sum_{m=1}^{p-1} \frac{\chi(m)}{A^{k}(m, p)}=0
$$

This proves Lemma 3.
Lemma 4. Let $p$ be a prime with $p \equiv 1 \bmod 3$ and $\lambda$ be any third-order character modulo $p$. Then, for any positive integer $k$, we have the identity

$$
\sum_{m=1}^{p-1} \frac{\lambda(m)+\bar{\lambda}(m)}{A^{k}(m, p)}=\frac{p-1}{A^{k}(1, p)}-H(k, p) .
$$

Proof. From the properties of the third-order character modulo $p$, we have

$$
1+\lambda(m)+\bar{\lambda}(m)= \begin{cases}3, & \text { if } m \text { is } a 3-\text { the residue modulo } p \\ 0, & \text { otherwise. }\end{cases}
$$

From (32), the properties of the 3 -th residue modulo $p$, and the identity $A^{k}\left(m^{3}, p\right)=A^{k}(1, p)$ where $(m, p)=1$, we have

$$
\begin{aligned}
\sum_{m=1}^{p-1} \frac{\lambda(m)+\bar{\lambda}(m)}{A^{k}(m, p)} & =\sum_{m=1}^{p-1} \frac{1+\lambda(m)+\bar{\lambda}(m)}{A^{k}(m, p)}-\sum_{m=1}^{p-1} \frac{1}{A^{k}(m, p)} \\
& =\sum_{m=1}^{p-1} \frac{1}{A^{k}\left(m^{3}, p\right)}-\sum_{m=1}^{p-1} \frac{1}{A^{k}(m, p)}=\frac{p-1}{A^{k}(1, p)}-H(k, p) .
\end{aligned}
$$

This proves Lemma 4. 
Lemma 5. Let $q>2$ be an integer; then, for any integer a with $(a, q)=1$, we have the identity

$$
S(a, q)=\frac{1}{\pi^{2} q} \sum_{d \mid q} \frac{d^{2}}{\phi(d)} \sum_{\substack{\chi \bmod d \\ \chi(-1)=-1}} \chi(a)|L(1, \chi)|^{2},
$$

where $L(s, \chi)$ denotes the Dirichlet L-function corresponding to the character $\chi$ and $\sum \chi \bmod p$ denotes the summation over all odd characters modulo $d$.

Proof. See Lemma 2 of [14].

\section{Proofs of the Theorems}

In this section, we shall complete the proofs of our all theorems. If $p$ be a prime, then from Lemma 5, we have

$$
S(a, p)=\frac{1}{\pi^{2}} \cdot \frac{p}{p-1} \cdot \sum_{\substack{\chi \bmod p \\ \chi(-1)=-1}} \chi(a)|L(1, \chi)|^{2} .
$$

It is clear that if $h$ is an integer with $(2, h)=1$, then note that the identities $S^{h}(-m, p)=-S^{h}(m, p)$ and $A(-m, p)=A(m, p)$, and we have

$$
\sum_{m=1}^{p-1} \frac{S^{h}(m, p)}{A^{k}(m, p)}=\sum_{m=1}^{p-1} \frac{S^{h}(-m, p)}{A^{k}(-m, p)}=-\sum_{m=1}^{p-1} \frac{S^{h}(m, p)}{A^{k}(m, p)}=0
$$

Now, for any positive integer $k$, from (35), we have the identity

$$
\sum_{m=1}^{p-1} \frac{S\left(m^{2}, p\right)}{A^{k}(m, p)}=\frac{1}{\pi^{2}} \cdot \frac{p}{p-1} \cdot \sum_{\substack{\chi \bmod p \\ \chi(-1)=-1}} \sum_{m=1}^{p-1} \frac{\chi^{2}(m)}{A^{k}(m, p)}|L(1, \chi)|^{2} .
$$

If $p=12 h+1$, then for any odd character $\chi \bmod p, \chi^{6}=$ $\chi_{0}$ if and only if $\chi=\chi_{0}, \chi_{2}, \chi_{2} \lambda$ or $\chi_{2} \lambda^{2}$, where $\chi_{2}$ is Legendre's symbol modulo $p$. In this time, $\chi$ is not an odd character modulo $p$. So, from (37) and Lemma 3, we have

$$
\sum_{m=1}^{p-1} \frac{S\left(m^{2}, p\right)}{A^{k}(m, p)}=0
$$

If $p=12 h+7$, then for any odd character $\chi \bmod p, \chi^{6}=$ $\chi_{0}$ if and only if $\chi=\chi_{2}, \chi_{2} \lambda$ or $\chi=\chi_{2} \bar{\lambda}$. So, in this time, note that $\left|L\left(1, \chi_{2} \lambda\right)\right|^{2}=\left|L\left(1, \chi_{2} \bar{\lambda}\right)\right|^{2}$; applying (37) and Lemma 4, we have

$$
\begin{aligned}
\sum_{m=1}^{p-1} \frac{S\left(m^{2}, p\right)}{A^{k}(m, p)} & =\frac{1}{\pi^{2}} \cdot \frac{p}{p-1} \cdot\left(\sum_{m=1}^{p-1} \frac{\lambda(m)}{A^{k}(m, p)}+\sum_{m=1}^{p-1} \frac{\bar{\lambda}(m)}{A^{k}(m, p)}\right) \cdot\left|L\left(1, \chi_{2} \lambda\right)\right|^{2}+\frac{h_{p}^{2}}{p-1} \sum_{m=1}^{p-1} \frac{1}{A^{k}(m, p)} \\
& =\frac{1}{\pi^{2}} \cdot \frac{p}{p-1} \cdot\left(\frac{p-1}{A^{k}(1, p)}-H(k, p)\right) \cdot\left|L\left(1, \chi_{2} \lambda\right)\right|^{2}+\frac{h_{p}^{2}}{p-1} \cdot H(k, p)
\end{aligned}
$$

where $\lambda$ is any third-order character modulo $p$.

It is clear that Theorem 1 follows from (36) and (38).

Theorem 2 follows from (39) and Lemma 2.

Now, we prove Theorem 3 . Note that $p \equiv 1 \bmod 3$ and (see [17] or [18]):

$$
\begin{gathered}
\sum_{\substack{\chi \bmod p \\
\chi(-1)=-1}}|L(1, \chi)|^{4}=\frac{5 \pi^{4}}{144} \cdot p+O\left(\exp \left(\frac{4 \ln p}{\ln \ln p}\right)\right), \\
\sum_{\substack{\chi \bmod p \\
\chi(-1)=-1}}|L(1, \chi)|^{2} \cdot|L(1, \chi \lambda)|^{2}=\frac{p}{2} \cdot \sum_{n=1}^{\infty} \frac{\left|\sum_{d \mid n} \lambda(d)\right|^{2}}{n^{2}}+O\left(\exp \left(\frac{4 \ln p}{\ln \ln p}\right)\right) .
\end{gathered}
$$

From (35), (40), and (41) and Lemma 3, we have the asymptotic formula as follows: 


$$
\begin{aligned}
\sum_{m=1}^{p-1} \frac{S^{2}(m, p)}{A^{k}(m, p)} & =\frac{1}{\pi^{4}} \frac{p^{2}}{(p-1)^{2}} \sum_{\substack{\chi \bmod p \\
\chi(-1)=-1}} \sum_{\substack{\chi \bmod p \\
\chi(-1)=-1}} \sum_{m=1}^{p-1} \frac{\chi(m) \eta(m)}{A^{k}(m, p)}|L(1, \chi)|^{2}|L(1, \eta)|^{2} \\
& =\frac{1}{\pi^{4}} \cdot \frac{p^{2}}{(p-1)^{2}} \cdot\left(\sum_{m=1}^{p-1} \frac{1}{A^{k}(m, p)}\right) \cdot \sum_{\substack{\chi \bmod p \\
\chi(-1)=-1}}\left(|L(1, \chi)|^{4}+2|L(1, \chi)|^{2}|L(1, \chi \lambda)|^{2}\right), \\
& =\left(\frac{5 p}{144}+\frac{p}{\pi^{4}} \cdot \sum_{n=1}^{\infty} \frac{\left|\sum_{d \mid n} \lambda(d)\right|^{2}}{n^{2}}\right) \cdot H(k, p)+O\left(|H(k, p)| \cdot \exp \left(\frac{4 \ln p}{\ln \ln p}\right)\right) .
\end{aligned}
$$

Applying Lemma 2 and mathematical induction, we can easily deduce the estimate

$$
|H(k, p)| \ll \frac{p}{|d|^{k}} .
$$

In fact, from Lemma 2 , we have $H(0, p)=p-1$, $H(1, p)=-p-1 / d$, and $H(2, p)=3(p-1) / d^{2}$. So, (43) is true for $k=0,1$, and 2 . Note that the third-order linear recursive formula is as follows:

$$
H(k, p)=-\frac{3}{d} H(k-1, p)+\frac{1}{d p} H(k-3, p) .
$$

So, for $k \geq 3$, from the mathematical induction, we can easily deduce the estimate

$$
|H(k, p)| \leq \frac{3}{|d|} \cdot|H(k-1, p)|+\frac{1}{|d| \cdot p} \cdot|H(k-3, p)| \ll \frac{p}{|d|^{k}} .
$$

Combining (42) and (43), we complete the proof of Theorem 3.

\section{Conclusion}

The main results in this paper are three theorems, which are closely related to Dedekind sums and cubic Gauss sums. They describe that when $p$ is a prime, the hybrid power mean of the Dedekind sums and cubic Gauss sums has good mean distribution properties. In fact, we can give some exact calculating formulae (see Theorems 1 and 2) or asymptotic formula (see Theorem 3) for them.

\section{Data Availability}

The data generated or analysed during this study are included in this published article.

\section{Conflicts of Interest}

The authors declare that there are no conflicts of interest regarding the publication of this paper.

\section{Authors' Contributions}

All authors have equally contributed to this work. All authors read and approved the final manuscript.

\section{Acknowledgments}

This work was supported by the N. S. F. of P. R. China (12161031), the N. S. F. of Inner Mongolia (CN) (2021MS01003), and the Number Theory Research Center of Hetao College.

\section{References}

[1] J. Greene and D. Stanton, "The triplication formula for Gauss sums," Aequationes Mathematicae, vol. 30, no. 1, pp. 134-141, 1986.

[2] W. P. Zhang and J. Y. Hu, "The number of solutions of the diagonal cubic congruence equation mod $p$," Mathematical Reports, vol. 20, pp. 70-76, 2018.

[3] L. Chen and J. Y. Hu, "A linear recurrence formula involving cubic Gauss sums and kloosterman sums," Acta Mathematica Sinica, vol. 61, pp. 67-72, 2018.

[4] X. X. Li and J. Y. Hu, "The hybrid power mean quartic Gauss sums and Kloosterman sums," Open Mathematics, vol. 15, pp. 151-156, 2017.

[5] S. F. Cao and T. T. Wang, "On the hybrid power mean of twoterm exponential sums and cubic Gauss sums," Journal of Mathematics, vol. 2021, Article ID 6638156, 8 pages, 2021.

[6] S. Chowla, J. Cowles, and M. Cowles, "On the number of zeros of diagonal cubic forms," Journal of Number Theory, vol. 9, no. 4, pp. 502-506, 1977.

[7] T. M. Apostol, Modular Functions and Dirichlet Series in Number Theory, Springer-Verlag, Berlin, Germany, 1976.

[8] C. Jia, "On the mean value of Dedekind sums," Journal of Number Theory, vol. 87, no. 2, pp. 173-188, 2001.

[9] J. B. Conrey, E. Fransen, R. Klein, and C. Scott, "Mean values of Dedekind sums," Journal of Number Theory, vol. 56, no. 2, pp. 214-226, 1996.

[10] H. Rademacher, "On the transformation of $\log \eta(\tau)$," Journal of the Indian Mathematical Society, vol. 19, pp. 25-30, 1955.

[11] H. Rademacher, Dedekind Sums, Carus Mathematical Monographs, Math. Assoc. Amer., Washington DC, USA, 1972. 
[12] J. Li and H. Zhang, "Some identities related to Dedekind sums and the second-order linear recurrence polynomials," $A d$ vances in Difference Equations, vol. 2013, Article ID 2013-299, 1 page, 2013.

[13] D. Han and T. Wang, "On the Dedekind sums and two-term exponential sums," Chinese Annals of Mathematics, Series B, vol. 36, no. 4, pp. 603-612, 2015.

[14] W. Zhang, "On the mean values of Dedekind Sums," Journal de Théorie des Nombres de Bordeaux, vol. 8, no. 2, pp. 429-442, 1996.

[15] T. M. Apostol, Introduction to Analytic Number Theory, Springer-Verlag, Berlin, Germany, 1976.

[16] B. C. Berndt and R. J. Evans, "The determination of Gauss sums," Bulletin of the American Mathematical Society, vol. 5, no. 2, pp. 107-130, 1981.

[17] H. Ito, "An application of a product formula for the cubic Gauss sum," Journal of Number Theory, vol. 135, pp. 139-150, 2014.

[18] J. H. Li and D. Han, "Some estimate of character sums and its applications," Journal of Inequalities and Applications, vol. 328, no. 1, pp. 1-8, 2013. 